Cahiers de recherches médiévales

Journal of medieval studies

12 spécial | 2005

Une ville, une destinée : Orléans et Jeanne d'Arc

\title{
Personne, personnage
}

Jeanne d'Arc en France au XVII ${ }^{\mathrm{e}}$ siècle

Françoise Michaud-Fréjaville

\section{(2) OpenEdition \\ Journals}

Édition électronique

URL : https://journals.openedition.org/crm/736

DOI : $10.4000 / \mathrm{crm} .736$

ISSN : 1955-2424

Éditeur

Honoré Champion

\section{Édition imprimée}

Date de publication : 1 juin 2005

Pagination : 231-247

ISSN : $1272-9752$

Référence électronique

Françoise Michaud-Fréjaville, «Personne, personnage », Cahiers de recherches médiévales [En ligne], 12 spécial | 2005, mis en ligne le 28 juin 2008, consulté le 15 décembre 2022. URL : http:// journals.openedition.org/crm/736; DOI : https://doi.org/10.4000/crm.736 


\section{RM}

\section{$-20-$ \\ Personne, personnage : Jeanne d'Arc en France au XVII' ${ }^{\mathrm{e}}$ siècle}

L'auteur dramatique anglais Edward Bond le déclarait récemment, un de ses "grands désirs est d'empêcher que Jeanne d'Arc soit brûlée »' Les allusions à notre héroïne sont courantes dans la presse internationale, émises par les personnalités les plus inattendues, qui connaissent souvent son histoire mieux que maints journalistes et politiciens français. En France où elle fait parfois l'objet d'une franche détestation anticléricale, les théories les plus fantaisistes continuent à circuler sur son compte: Jeanne fille de Louis d'Orléans et d'Isabeau de Bavière, Jeanne hystérique ou sexuellement anormale, dépourvue de spiritualité, créature de Yolande l'Aragon ou de Regnault de Chartres, soustraite au bûcher avant le supplice ${ }^{2}$. Vraie ou fausse, Jeanne d'Arc nous est aujourd'hui si familière qu'on imagine mal un temps où elle aurait disparu du paysage quotidien. Mais les derniers siècles de l'Ancien Régime ne lui portèrent qu'un intérêt très inconstant ${ }^{3}$. Les pages qui lui furent alors consacrées ne méritant guère qu'on s'y attarde pour le plaisir, elles ont rebuté bon nombre de chercheurs. Il faut donc rendre grâce aux efforts de synthèses de P. Contamine ${ }^{4}$, d'A. Donot et Ann Powers ${ }^{5}$ qui ont abordé ces périodes obscures, tout comme au remarquable chapitre que Georges Huppert a consacré à l'historiographie de Jeanne d'Arc au XVI $\mathrm{e}^{\mathrm{e}}$ siècle dans L'idée de l'histoire parfaite ${ }^{6}$. Les auteurs du début de l'époque moderne se heurtaient en effet à une série de questions délicates : comment expliquer la condamnation et la mort de Jeanne sans mettre en accusation une Église implacable ou dans l'erreur, un roi de France trop timoré pour l'arracher à sa prison, voire jaloux de la gloire d'une simple paysanne? Reconnaître à la Pucelle un rôle trop éclatant dans l'expulsion des Anglais, c'était porter ombrage à Charles le Victorieux et ses successeurs, nier la validité des efforts militaires coûteux en hommes et en argent au profit d'une intervention divine difficilement reproductible. Il n'était guère opportun après 1525 , dans un pays bouleversé par la Réforme, de dénoncer

${ }^{1}$ Traduit dans Le Nouvel observateur, 19-25 novembre 1998, p. 150.

${ }^{2}$ Y. Grandeau relève et réfute la plupart de ces théories extrémistes dans Jeanne insultée. Procès en diffamation, Paris, 1973.

${ }^{3} \mathrm{P}$. Marot, «De la réhabilitation à la glorification de Jeanne d'Arc. Essai sur l'historiographie et le culte de l'héroïne en France pendant cinq siècles », dans Mémorial du $V^{e}$ centenaire de Jeanne d'Arc, 1436-1956, Paris, 1958.

${ }^{4} \mathrm{P}$. Contamine, « Naissance d'une historiographie. Le souvenir de Jeanne d'Arc, en France et hors de France, depuis le «Procès de son innocence » (1453-1456) jusqu'au début du XVI ${ }^{\mathrm{e}}$ siècle », Francia, 15 (1987), p. 233-256 ; Id., «Henry VI, première partie, la Guerre de Cent ans, Jeanne d'Arc », dans M.-Th. Jones-Davies éd., Shakespeare et la Guerre, actes du colloque de la Société française Shakespeare 1989, Paris, 1990, p. 113-127.

${ }^{5}$ A Donot, «Jeanne d'Arc dans la littérature du XVII siècle », dans Dictionnaire des Lettres françaises : XVII siècle, Paris, 1954. On doit regretter que le travail de A. B. Powers, The image of Jeanne d'Arc in seventeenth-century France, Ph. D., University of Cincinnati, 1967, ne soit pas mieux connu en France.

${ }^{6} \mathrm{G}$. Huppert, L'idée de l'histoire parfaite, Paris, 1972, appendice III : « Jeanne d'Arc, le dossier historiographique », p. 204-216.

Cahiers de Recherches Médiévales (XII $-X V^{e}$ s.), 12spé, 2005 
trop fortement une assemblée de clercs capable de se tromper et de tromper une innocente. Évoquer simplement les arguments suggérant que Jeanne était une sorcière, tout en glorifiant la jeune fille, allait à l'encontre du grand mouvement de chasse aux possédées du diable et de la dénonciation généralisée du moindre soupçon de pratiques occultes. Comme le suggère si joliment G. Huppert, pour les écrivains d'alors «moins ils en diraient, mieux ce serait».

Cependant, tout n'est pas à rejeter pour l'historiographie dans les travaux souvent besogneux des auteurs classiques, et je me propose ici de revenir sur quelques œuvres du XVII ${ }^{\mathrm{e}}$ siècle dont Jeanne est l'héroïne. Nous n'évoquerons que très succinctement la tragédie de Virey des Graviers, jouée à Rouen en $1600^{7}$, pour nous attarder sur les suivantes. Johanna Darcia, une tragédie en vers latins de Nicolas de Vernulz (1629) ${ }^{8}$ est pratiquement inconnue aujourd'hui, et son auteur totalement oublié. En deux versions aussi médiocres l'une que l'autre, la tragédie de La Pucelle d'Orléans de François d'Aubignac ${ }^{9}$ et son double versifié par La Ménardière (1642) seront ensuite abordées ${ }^{10}$. Nous terminerons par La Pucelle de Jean Chapelain $(1656)^{11}$, qui figure dans toutes les bibliographies johanniques, en général de peu flatteuse manière. Cependant, avant d'examiner non pas les caractères littéraires de ces textes mais leur intérêt pour l'histoire du «sentiment johannique $»^{12}$, car je rappelle que je me place en historienne médiéviste, il m'a paru nécessaire dans un premier temps de me pencher sur la littérature concernant l'héroïne qui pouvait être accessible à nos auteurs.

Philippe Contamine a parfaitement relevé, dans les dernières décennies du Moyen Âge, un intérêt particulier pour la compilation par des abréviateurs des textes des procès présentés en des ouvrages d'utilisation aisée ${ }^{13}$. L'abbaye de Saint-Victor de Paris, entre autres, permettait libéralement aux érudits et amateurs l'accès à ces breviarum. Grâce à l'imprimerie, le Champion des dames de Martin Le Franc ${ }^{14}$ où

${ }^{7}$ J. de Viray des Graviers, Tragédie de Jeanne Darques dite la Pucelle d'Orléans, native du village d'Emprenne près Vaucouleurs en Lorraine, Rouen, Raphael de Petitval [1600].

${ }^{8}$ Nicolai Vernulxi, publici eloquentice professoris in Academia Lovanensis, Joanna Darcia, vulgo Puella Aurelianensis, tragedia, Lovanii, typ. Phil. Dormalii, 1629. L'œuvre eut deux autres éditions dans les recueils de ce professeur d'éloquence, en 1631 et 1656 ; je la cite d'après Jeanne d'Arc, tragédie latine en cinq actes par Nicolas de Vernulz, accompagnée d'une traduction en regard et d'une dédicace-introduction, éd. A. de Latour, Orléans, Herluison, 1880 . Cette édition ne fut tirée qu'à 260 exemplaires.

${ }^{9}$ F. Hédelin, abbé d'Aubignac, La Pucelle d'Orléans, tragédie, Paris, François Targa, 1642.

${ }^{10}$ [La Mesnardière], La Pucelle d'Orléans, tragédie, Paris, Anthoine de Sommaville et Augustin Courbé, 1642.

${ }^{11}$ J. Chapelain, La Pucelle ou la France délivrée, Paris, Augustin Courbé, 1656 pour les douze premiers chants, et La Pucelle, chants XII à XXIV, éd. R. Kerviler, Orléans, Herluison, 1882 (tirage à 26 exemplaires !).

${ }^{12}$ Voir infra, articles n ${ }^{\circ} 21$ à 24.

${ }^{13}$ P. Contamine, « Naissance d'une historiographie », op. cit., p. 254-256.

${ }^{14}$ Martin le Franc, Le champion des dames, Lyon, Guillaume Le Roy, [v. 1485] ; édition Paris, Galiot Dupré, 1530. Il n'en subsiste que six manuscrits (depuis 1440 jusqu'à la fin du XV ${ }^{\mathrm{e}}$ siècle) dont un est passé récemment en vente publique. Depuis la rédaction de cet article, une édition critique du Champion des dames a été faite par R. Deschaux (5 vol., Paris, Champion, CFMA 127-131, 1999). 
Jeanne était apparue dès 1440 au nombre des femmes célèbres et surtout les Vigiles de Charles VII de Martial d'Auvergne ${ }^{15}$, une chronique des victoires royales accompagnée de gravures montrant les épisodes marquants du règne, ont favorisé une relative vulgarisation du personnage de Jeanne. Contamine suggérait également que l'élévation au trône de Louis XII avait peut-être encouragé la parution de textes rappelant discrètement la fidélité de la famille d'Orléans à la cause de Charles VII ${ }^{16}$. J'ajouterai, pour le temps de François $1^{\mathrm{er}}$, l'effet d'amplification, la valeur d'exemple déjà ancien et le rôle attribué désormais à la loi salique dans la dévolution si particulière du pouvoir royal en France : tout ce qui confortait la continuité royale par les mâles méritait de rester en mémoire.

La vogue des écrits spécifiquement consacrés à la Pucelle ne se prolonge guère dans un premier temps au-delà de 1525 , comme le montre la recension minutieuse des ouvrages historiques et littéraires ayant contribué à la connaissance de la Pucelle établie en 1894 par Lanéry d'Arc ${ }^{17}$, enrichie de notes marginales dues aux conservateurs successifs du Musée historique d'Orléans puis du Centre Jeanne d'Arc. La personne même de Jeanne d'Arc n'est plus au cœur des publications, même si elle est souvent évoquée. La Pucelle n'occupe déjà plus qu'un folio sur les cinq cent trente et quelques que comportent les deux tomes de la Mer des hystoires ${ }^{18}$. Le plus grand succès historique du $\mathrm{XV}^{\mathrm{e}}$ siècle, le Compendium de Robert Gaguin $(† 1503)$, avait connu au moins huit éditions latines de 1495 à 1514 , suivies d'une douzaine en français jusqu'en 1536 sous le titre de Mer des croniques et Miroir historial $^{19}$, dont la partie sur Jeanne d'Arc fut largement reprise par Baptiste Fulgo-

${ }^{15}$ [Martial d'Auvergne], S'ensuivent les vigilles de la mort du feu roi Charles septiesme à neuf pseaulmes et sept leçons, contenant la cronique et les faits advenuz durant la vie du dit feu roy, composées par Maistre Martial de Paris, dit d'Auvergne, procureur en parlement, [Paris, Jehan Dupré, 1493. Ppas moins de sept éditions sont repérées jusqu'en 1505. Le manuscrit de présentation à Charles VIII (1484) est le BnF, fr. 9677, dont les miniatures, avec une Jeanne en jupe et cheveux longs, sont fort célèbres.

${ }^{16}$ Voir A. W. Lewis, Le sang royal. La famille capétienne et l'État, France, XI $-X I V^{e}$ siècles, Paris, 1986, p. 201.

${ }^{17} \mathrm{P}$. Lanéry d'Arc, Le Livre d'or de Jeanne d'Arc. Bibliographie raisonnée et analytique des ouvrages relatifs à Jeanne d'Arc. Catalogue méthodique, descriptif et critique des principales études historiques, littéraires et artistiques consacrées à la Pucelle d'Orléans depuis le XV siècle jusqu'à nos jours. Paris, 1894, 1008 pages. L'ouvrage offre 2127 précieuses notices. On consultera également avec fruit N. Margolis, Joan of Arc in History, Litterature and Film. A select annotated bibliography, New-York-Londres, 1990, particulièrement les p. 265-293.

${ }^{18}$ La Mer des hystoires, Paris, Vincent Comin et Pierre Lerouge 1488, deux grands in-folio sur deux colonnes, le passage sur Jeanne d'Arc se trouve au t. II, fol. 238v-239v (voir Lanéry d'Arc, op. cit., p. 28, n $\left.{ }^{\circ} 27\right)$.

${ }^{19}$ Robert Gaguin, Compendium de origine et gestis Francorum, Paris, Pierre Le Dru, 1495. Le Miroir historial originel est une compilation anonyme (par Noël de Fribois ?), commande royale de 1451 qui s'arrêtait en 1380. [R. Gaguin], Les Chroniques de France ou Mirouer historial de France, traduites par Pierre Desrey, Paris, Poncet le Preux et Galiot Du Pré, 1514 , ont été suivies de nombreuses rééditions chez divers imprimeurs. Voir F. Collard, Un historien au travail à la fin du XV $V^{e}$ siècle : Robert Gaguin, Genève, 1996 ; Id., " Histoire de France en latin et histoire de France en langue vulgaire : la traduction du Compendium de origine et gestis Francorum de Robert Gaguin au début du XVI ${ }^{\mathrm{e}}$ siècle », dans Histoires de France, historiens de la France. Actes du colloque international, Reims, 14 et 15 mai 1993, Y.-M. Bercé et P. Contamine éd., Paris, 1994, p. 91-118. 
$\mathrm{si}^{20}$. Les Annales de Nicole Gilles ( $\left.\dagger 1503\right)$, considérées comme plus modernes et imprimées dès 1492 , traversent le $\mathrm{XVI}^{\mathrm{e}}$ siècle avec de constantes actualisations à partir de $1525^{21}$, entre autres par Belleforest qui ajoute beaucoup de sa plume à la geste de la Pucelle en consultant les procès ${ }^{22}$. Monstrelet demeure, à travers de nombreuses réimpressions, le seul ou presque des auteurs hostiles Jeanne dont l'œuvre soit vraiment répandue et souvent utilisée, davantage sans doute pour son talent d'écrivain que pour son traitement fort critiques du personnage ${ }^{23}$.

Jeanne d'Arc apparaît aussi, par choix délibéré des auteurs ou compilateurs et non en raison de son rôle historique dans tous les recueils de vies illustres, sous des prétextes et des titres divers: Le Mirouer des femmes vertueuses faussement donné à Alain Bouchard ${ }^{24}$, le De plurimis claris scelestique mulieribus... de Jacques-Philippe Foresti de Bergame $(1497)^{25}$, repris par Ravisius Textor en $1521^{26}$, ou la Nef des dames vertueuses de Champier ${ }^{27}$. La Pucelle y est généralement en compagnie des femmes fortes de la Bible, Déborah et Judith, auxquelles tous ses défenseurs l'avaient comparée lors de l'enquête de réhabilitation, ou des héroïnes antiques de l'histoire chères à ses premiers biographes, l'amazone Penthésilée, les reines Sémi-

\footnotetext{
${ }^{20}$ Battista Fulgosi, De dictis factisque memorabilibus collecteana, a Camilla Gilina latina facta, Milan, Jacques Ferrarius, 1509 ; et Paris, Galiot du Pré, 1518.

${ }^{21}$ Nicole Gilles, Les tres elegantes, tres veridiques et copieuses annales des tres preux, tres nobles [...] compilees par feu maistre Nicole Gilles, Paris, Galiot du Pré, 1525.

${ }^{22}$ [Nicole Gilles], Les chroniques et annales de France, des origines des françoys et leur venues es Gaules faites jadis briesvement par Nicole Gilles secrétaire du roy jusqu'au roi Charles huitiesme et depuis additionnees par Denis Sauvage jusqu'au roy François second du nom, revu par Belleforest et Chappuys, Paris, Jean Cavellat, 1585. Le texte de Belleforest sur l'épopée de Jeanne se trouve aux fol. 309-328v, le procès en nullité est largement cité aux fol. 359-366v.

${ }^{23}$ Enguerrand de Monstrelet, Les chroniques d'Angleterre et de Bourgogne, Paris, A. Vérard, [v. 1490]. Ces chroniques eurent au moins trois autres éditions jusqu'en 1518 (Vérard, Le Noir, Regnault).

${ }^{24}$ Alain Bouchard, Le mirouer des femmes vertueuses, ensemble la patience Griselidis. L'histoire admirable de Jehanne pucelle de Veaucouleurs. L'édition la plus ancienne repérée par Lanéry d'Arc était de Lyon, chez feu Barnabé Chaussard, 1546, mais on sait qu'Alain Bouchard a fini sa carrière comme secrétaire de Charles VIII et que ses Grandes chroniques de Bretaigne ont été imprimées en 1514 (E. Port, «Alain Bouchard, chroniqueur de Bretagne », Annales de Bretagne, 1926, p. 496-527, et 1925, p. 68-601). En fait, l'ouvrage de 1546 réunit des œuvres différentes, mais l'opposition est intéressante entre Grisélidis, la mère résignée, et Jeanne, la vierge battante (cf. N. Margolis, op. cit., p. 104, notice 357).

${ }^{25}$ Jacques-Philippe Foresti de Bergame, De plurimis clarisque scelestique mulieribus. Opus prope divinis novissima congestum [écrit vers 1493, première impression connue: Ferrare, 1497].

${ }^{26}$ Ravisius Textor (Jean Tixier de Ravisì), professeur au collège de Navarre, De memorabilibus et claris mulieribus aliquot diversorum scriptorum opera, Paris, Simon de Colines, 1521. Il donne à la suite du texte de Jacques-Philippe de Bergame (De claris mulieribus) le poème de Valeran de la Varanne, De gestis Ioanne virginis France Anglorum expultricis, egregie bellatricis, libri quatuor, écrit dans la seconde moitié du XV siècle et imprimé pour la première fois à Paris chez Jean de la Porte [1516].

${ }^{27}$ Symphorien Champier, La Nef des dames vertueuses composée par maistre Simphorien Champier docteur en médecine; Lyon, Jacques Arnollet [1503], qui fut suivi de deux éditions parisiennes en 1515 et 1531 .
} 
ramis, et Zénobie, Clélie, jeune otage en fuite, graciée par le roi étrusque Porsenna après une aventureuse traversée du Tibre à la nage, et qui fut statufiée par les Romains sur le cheval offert par le roi dont elle avait forcé l'admiration ${ }^{28}$. L'historien humaniste Polydore Virgile évoquait la magnanimité de Porsenna en relatant la fin cruelle de Jeanne ${ }^{29}$, mais le détail de la statue équestre ne pouvait pourtant pas relier directement cet épisode romain aux premiers monuments élevés au XVI ${ }^{\mathrm{e}}$ siècle à la Pucelle, tant à Orléans qu'à Rouen, car Jeanne n'y était pas figurée en cavalière, modèle apparu au milieu du $\mathrm{XIX}^{\mathrm{e}}$ siècle $^{30}$.

On voit ainsi dès le premier quart du XVI ${ }^{\mathrm{e}}$ siècle se dessiner les deux grands types de littérature - histoire générale et vies illustres - auxquels vont avoir recours les nouveaux historiens de Jeanne d'Arc pour compiler leurs ouvrages en un genre resté très médiéval : le florilège. Trois recueils parus entre 1576 et 1645 offrent un bouquet des extraits que leurs auteurs jugeaient significatifs pour illustrer leur propos en l'honneur de Jeanne d'Arc. En 1576 on put lire l'Histoire et discours au vray du siège qui fut mis devant la ville d'Orléans de Léon Tripault ${ }^{31}$, qui n'était en fait que l'impression d'un ouvrage antérieur. Les Comptes de ville d'Orléans pour l'année 1468 signalent la commande par le corps municipal orléanais à un certain Pierre Soudan d'une histoire du siège, destinée aux archives de la ville. La décision des échevins de la faire imprimer avec la caution d'un conseiller connu, en plein cœur des guerres de religion, et son succès immédiat s'expliquent par valeur retrouvée du symbole, mutatis mutandis, après un nouveau siège de la cité (1563) et la reprise en main de la ville par le parti royal et légaliste de Catherine de Médicis ${ }^{32}$. Sa fortune ultérieure, avec de multiples rééditions plus ou moins illustrées et augmentées, est due à ses qualités de témoignage proche, son parfum d'authenticité, car le véritable rédacteur avait eu au milieu du $X V^{\mathrm{e}}$ siècle accès à des témoignages tout à fait contemporains des événements. Trente-six ans plus tard Jean Hordal proposait à son tour une Heroinae nobilissimae Iannae Darc ... historia... ${ }^{33}$ qui deviendra la source fondamentale des écrivains du XVII ${ }^{\mathrm{e}}$ siècle grâce aux extraits et citations d'ouvrages sur la Pucelle qu'elle leur offrait. Enfin, en 1645 François Lahier faisait

\footnotetext{
${ }^{28}$ Étienne Forcatule énumère également la mère de Darius, celle des frères Macchabées et, en prime, Camille princesse guerrière volsque (d'après Virgile, Enéide, VII).

${ }^{29}$ The tree Books of Polydore Vergil's English History, an early translation, preserved among the mss of the old Royal library in the British Museum, Sir Henry Ellis éd., 1844, p. 38.

${ }^{30}$ Avec Foyatier à Orléans, 1855.

${ }^{31}$ [Léon Tripault], L'histoire et discours au vray du siege qui fut mis devant la ville d'Orléans par les Anglais, le mardi 12 jour d'octobre 1428, regnant alors Charles VII, roi de France, contenant toutes les saillies, assaults, escarmouches et autres particularitez notables qui de jour en jour y furent faictes, avec la venue de Jeanne la Pucelle[...] par M. Léon Tripault, conseiller en icelle ville, Paris, Saturny Hottot, 1576. In $8^{\circ}, 4+50$ fol.

${ }^{32}$ En 1576, Catherine de Médicis offrit à la cathédrale Sainte-Croix, presque entièrement détruite par les Protestants en 1562, des reliques de la vraie croix, son fils Henri d'Anjou (futur Henri III) fit cette même année un séjour à Orléans.

${ }^{33}$ [Jean Hordal], Heroinae nobilissimae Iannae Darc Lotharingiae vulgo aureliaensis puellae historia, ex variis gravissimae atque incoruptissimae fidei scriptoribus excerpta ejusdem mavortiae virginis innocentia a calumniis vindicata, authore Ioanne Hordal serenissimi ducis lotharingiae consiliario et I[n] V[traque] doctore ac professore publico in alma universitate Ponti-Mussana, Pont-à-Mousson, Melchior Bernard, 1612, In 8, IX-251p.
} 
imprimer le Grand Ménologe des saintes... ${ }^{34}$ dont la notice concernant Jeanne paraît ouvrir l'ère fort longue de la procédure de canonisation.

Il semble très légitime de s'attacher un moment au travail de Jean Hordal et à sa descendance directe, une tragédie en latin signée Nicolas Vernulz. Nul ne lit plus aujourd'hui la compilation d'Hordal qui ne fut jamais traduite alors que les gravures originales de Léonard Gaultier ont été souvent imitées, encore plus souvent découpées et vendues à part, au grand dam de la conservation du texte. Le frontispice représente le monument du pont d'Orléans dans sa version postérieure à 1562 - date de sa première destruction par les protestants -, la page 2 verso montre Jeanne d'Arc à cheval Talis in arma ruit bellaci scemate virgo, tandis que le privilège offre la célèbre Jeanne dite «des échevins », en chapeau à panache et robe, l'épée dressée de la main droite et le mouchoir tenu de la gauche, Talis inermis erat muliebri veste puella $^{35}$. Le grand nombre de ces gravures isolées atteste de la véritable popularité d'un livre dont la langue n'était pas si difficile pour l'honnête homme et qui offrait une série de fort précieuses références.

Conseiller d'État de Lorraine et professeur à l'université de Pont-à-Mousson, Hordal appartient, nous dit-il lui-même longuement, à la famille de la Pucelle par son frère Pierre d'Arc, ce qui peut expliquer l'intérêt qu'il lui porte. Il a dédié tout naturellement et habilement son œuvre à François de Lorraine, comte de Salm et de Vaudémont, mais surtout seigneur de Domrémy. On peut diviser le livre en trois parties: 1'histoire de la Pucelle jusqu'à sa capture à Compiègne (25 pages) et le procès avec l'apologie de l'«amazone chrétienne» (17 pages) encadrent une copieuse suite de citations classées par grands genres, suivie d'une liste non systématiquement récapitulative de titres et d'auteurs (122 pages). L'essentiel du travail est donc une accumulation de textes, un de ces florilèges d'arguments et d'exemples comme les auteurs de sermons et les universitaires en utilisaient depuis des siècles. Si l'on prend le soin de regarder de près cette énumération on constate que la bibliothèque de l'Université lorraine, complétée sans doute par la collection propre des Hordal, disposait de ce que nous appellerions aujourd'hui un «beau fonds johannique .

Pour résumer, et nous suivrons l'ordre qu'il donne, Hordal cite quinze théologiens, six juristes, deux médecins, vingt-cinq historiens, quatre poètes, puis à ces cinquante-deux références plura hujus historice firmamenta ac testimonia, si quis requirat, consulat, il ajoute une liste de cinquante noms et titres dont certains sont des redites des précédentes énumérations. Il ne se contente pas - fort heureusement de simples titres ou des mentions brèves : quelques longs extraits, par exemple celui de Robert Gaguin (p. 66-87), reprennent assez fidèlement le texte, facile à retrouver puisque les références sont généralement exactes; de même pour Philippe de Bergame (p. 40-50), ou Jacques Meyer (p. 57-65) ${ }^{36}$. Il est plus difficile de vérifier le

${ }^{34} \mathrm{~F}$. Lahier, Le grand Ménologe des saintes, Bien-heureuses et Vénérables vierges contenant [...] les éloges de celles qui ont vécu au monde en l'éminent état de la Virginité, Lille, Nicolas Rache, 1645 , In $4^{\circ}, 611+560 \mathrm{p}$.

${ }^{35}$ Hordal, p. 2v. : «Ainsi la vierge combattait en costume de guerre »; et au privilège : « Ainsi désarmée était vêtue la Pucelle en vêtement de femme ».

${ }^{36}$ Jacques Meyer, Commentarius sive annales rerum flandricarum, Anvers, J. Steel, 1561. 
long passage attribué à Etienne Forcatule, docteur de l'Université de Toulouse ${ }^{37}$ (p. 99-111), qui rapporte, à propos du monument élevé sur le pont d'Orléans en l'honneur de la Pucelle, où cette dernière n'était pourtant pas en cavalière, l'anecdote de Clélie Porsenna merito reditta évoquée plus haut. Les morceaux choisis par Hordal ouvrent un large éventail de références qu'il est trop long d'étudier ici pièce à pièce, - travail qui serait, je crois, fort profitable à l'historiographie française et pas seulement à celle de Jeanne. On pourrait également relever le rôle non négligeable que joua la toute jeune université de Pont-à-Mousson, née en 1573, dans la perpétuation de la mémoire de la Pucelle. Je me contenterai à ce propos de faire quelques remarques: on a la quasi la certitude qu'Hordal, comme plus tard Denis Godefroy, a consulté et copié largement un texte du procès de réhabilitation ${ }^{38}$. De plus, une bonne partie des auteurs qu'il cite, soit Meyer, saint Antonin de Florence, Gilbert Genebrard, Polydore Vergil, les plus anciens et qui paraissaient les plus difficiles à consulter à l'exception de Gaguin, se trouvent en réalité réunis dans un manuscrit du XVI ${ }^{\mathrm{e}}$ siècle, actuellement à la Bibliothèque Nationale de France, qui comprend une douzaine des argumentaires en faveur de l'innocence de Jeanne d'Arc : Innocentia et fortitudo puella .... ${ }^{39}$. Ce manuscrit - ou une copie - se trouvaitil à Pont-à-Mousson ? À ces textes, déjà anciens à l'époque, il faut ajouter une bibliographie alors moderne dont les ouvrages cités devaient certainement se trouver dans cette Université des jésuites de Lorraine. Hordal cite les œuvres des deux frères du Tillet (†1570) Jean l'évêque de Meaux (page 134) pour sa Chronica de regibus francorum... parue en 1577, et l'autre Jean, greffier du Parlement de Paris, seigneur de la Bussière (page 135), de même que le Sommaire de l'histoire des François de Nicolas Vignier (1570) $)^{40}$ et les travaux du jésuite espagnol Juan de Mariana $(\dagger 1624)^{41}$. La bibliothèque comptait sans doute également l'Opus chronographium de Pierre Opmeer, dont Hordal cite un petit extrait à propos du pont d'Orléans, alors que la Chronographie n'était parue que l'année précédente ${ }^{42}$. Certes Hordal accumule les autorités sans grand esprit critique, et ne relève pas qu'elles se copient fortement les unes les autres. Il est vrai qu'à ses yeux ces ressemblances confirmaient, par la quantité, la validité des faits et le poids des anecdotes, telle la reconnaissance de Charles VII caché parmi les courtisans de Chinon teniore habitu inter regio cultu ornatos proceres (p. 14). Au long de sa dernière partie, pour son plaidoyer en faveur de la Pucelle, Hordal utilise très largement les arguments qu'il attri-

${ }^{37}$ Je soupçonne François Lahier d'avoir très largement pillé Hordal dans les extraits qu'il fournit à l'appui de sa vie de Jeanne d'Arc. Il le cite d'ailleurs parmi ses sources.

${ }^{38} \mathrm{La}$ sentence de « réhabilitation ou de nullité de la condamnation » est entièrement recopiée aux p. 194-205.

${ }^{39}$ Paris, BNF., ms fr. 20152. Le premier opuscule est la lettre de Guy de Laval en français, d'où le classement du recueil dans les «manuscrits français ».

${ }^{40}$ Hordal, p. 142. N. Vignier, Sommaire de l'Histoire des Français, Paris, 1570. Sur Vignier et Jeanne d'Arc, cf. G. Huppert, op. cit., p. 210.

${ }^{41}$ Hordal, p. 94-96. Juan de Mariana, Historia de rebus hispaniae, Tolède, P. Rodrigue, 1592, $2^{\mathrm{e}}$ éd. 1605 . Jean Mariana, jésuite qui avait été profès à Paris aurait pu y consulter le breviarum de Saint-Victor.

${ }^{42}$ Hordal, p. 140-142. P. Opmeer, Opus chronographicum orbis universi, Anvers, 1611. Hordal en cite une phrase assez passe-partout :[Johanna] Gallicas ducebat acies, mais il en extrait également une mention supplémentaire au monument d'Orléans: Gratitudinis ergo posuere Regi Joanne que aeras statuas in ponte ligeris S. P.Q. R. Aurelianus. 
bue, selon la tradition d'époque, à Gerson et d'Henri de Gorckum d'après la Sybilla francica seu de admirabili puelle $(1606)^{43}$.

Outre que Hordal s'intéressait personnellement à la défense et illustration de Jeanne d'Arc, sa présence à Pont-à-Mousson n'est pas étrangère à la documentation particulièrement abondante dont il a fait étalage. Une génération avant lui, Fronton du Duc avait montré que Jeanne n'était pas inconnue en ce lieu. L'histoire tragique de la Pucelle d'Orléans (qu'Hordal honore d'une citation) avait été jouée en 1581 devant Charles III de Lorraine, faute d'avoir pu l'être en présence du roi Henri III de France. L'auteur était un jeune professeur de rhétorique d'environ vingt-deux ans, qui par la suite se consacra entièrement à la théologie. Sa pièce est destinée à une représentation par les élèves, tous désireux sans doute de se montrer aux illustres spectateurs, ce qui explique le grand nombre de personnages - une trentaine. On peut s'interroger sur les raisons du choix d'un tel sujet. Voulait-on mettre en avant le rôle des femmes dans la sauvegarde du royaume ? L'éditeur de 1581, Jean Barnet, a glissé dans la préface, au contraire, de transparentes allusions aux malheurs actuels du royaume, c'est-à-dire la septième reprise des guerres de religion, et à cette reine de France qui voulait faire alliance avec un pays ennemi, soit Catherine et ses projets de marier son fils le duc d'Anjou avec la reine Elisabeth Tudor. Au cours de l'avant jeu de la pièce, l'archange saint Michel souligne dans un rappel des événements les risques d'une telle alliance ${ }^{44}$ :

[Le roi d'Angleterre] en poursuivant d'une fille le droict

Pensoit que des François par force il obtiendroit

Le sceptre tres sacré jusqu'à Charles sixieme

Qui sa fille donna pour femme au roy Anglois.

Mais cestuy tost après, méprisant toutes loix

Saisissant son beau Père affoibly de vieillesse

A Charles son beau frère ostat le droict d'aînesse

Se saisit de Paris...

L'action avance au pas de longues discussions. Elle mérite surtout notre attention par son second acte : le roi, le chancelier, l'évêque de Chartres (Robert Dauphin) et un docteur discutent de la mission de Jeanne en échangeant la plupart des arguments utilisés dans les deux procès. Le critique J.-J. Soons a minutieusement analysé les situations et les différents discours, recherchant les sources possibles. On

${ }^{43}$ Hordal, p. 50, donne d'abord le titre de: Libellus de Sybilla Franciae rotuli duo, puis cite nommément quelques pages plus loin le Propositionum de Puella militari d'Henri de Gorckum. Il disposait selon toute vraisemblance de l'édition de 1606 de la Sybilla francica, qui comprenait cinq petits opuscules du début du $\mathrm{XV}^{\mathrm{e}}$ siècle, dont les deux traités attribués à Gerson sur la Pucelle (cf. Lanéry d'Arc, op. cit., $\mathrm{n}^{\circ}$ 28). Voir N. Margolis, op. cit., p. 224-225, pour la question toujours controversée de nos jours de l'attribution des deux traités De mirabili victoria et De quadam puella.

${ }^{44}$ [Fronton du Duc], L'histoire tragique de la Pucelle de Dom-Remy autrement D'Orleans, nouvellement départie par actes et représentée par personnages, Nancy, veuve Jean Ianson, 1581 , et $I d$., publiée par Jean. Barnet, Pont - à-Mousson, Durand de Lançon, 1581. Sur cette œuvre: S. Beaudeuf, «L'Histoire tragique de la Pucelle de Domremy de Fronton du Duc, une œuvre politique de circonstance», Bulletin de la Société des Amis du Centre Jeanne d'Arc, $n^{\circ} 24,2000$, p. 87-115. 
ne sera pas surpris d'y trouver bon nombre des auteurs cités, donc après Fronton, par Hordal : l'Abréviateur de Saint-Victor (aujourd'hui dit le «manuscrit d'Orléans »), le procès de réhabilitation, Le Mirouer, Henri de Gorckum, Gerson, Monstrelet. Mais le commentateur y repère aussi la Chronique de la Pucelle et le Journal d'un bourgeois de Paris qu'Hordal n'a jamais donnés comme sources ${ }^{45}$. La personne de Jean d'Estivet au quatrième acte, premier acte de la captivité, est traitée avec une noirceur dont les chroniqueurs n'usent généralement pas, eux qui laissent les noms des juges de Rouen dans une ombre pudique :

Ne pensés point que ce soit une simplette femme

Mais ung corps qui en soy contient une telle ame

Qu'elle a en son noir sein occultement cachés

Les monstres venimeux des plus sales péchés $(\mathrm{IV}, 4)$

Au long de la pièce, Jeanne n'échange guère de propos avec les personnages historiques qui ne soient tirés des documents du $\mathrm{XV}^{\mathrm{e}}$ siècle ${ }^{46}$, garantie donc de véracité. En revanche, dans ses monologues et certains des entretiens avec saint Michel, où l'imagination du dramaturge pouvait s'exercer, la jeune fille manifeste le caractère d'introspection des héroïnes des grandes tragédies classiques :

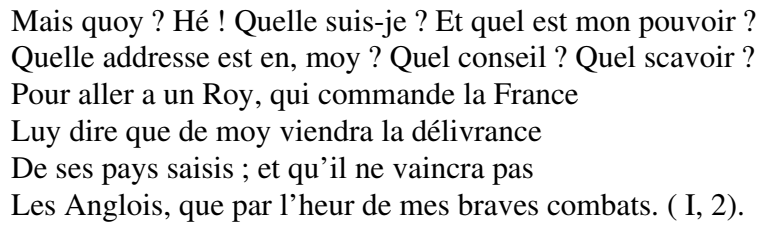

La fin de Jeanne n'est bien sûr pas présentée en scène, mais le récit d'un témoin rapporte les éléments merveilleux d'une légende déjà bien en place: le cœur de la Pucelle retrouvé encore saignant dans les braises, l'envol d'une colombe hors du brasier (autrement dit l'âme pure de la martyre) et enfin les cendres que de pieuses femmes ne purent recueillir et ensevelir parce que les bourreaux «les ont espars dans Seine par les flots ».

Les rares critiques qui se sont penchés sur ce texte ${ }^{47}$ y ont-ils repéré les caractères d'une œuvre «lorraine» de circonstance ? Dans la pièce, le roi René vient au conseil de Charles VII l'assurer de son appui et soutenir le parti de la Pucelle, alors qu'en 1580 la cour de Lorraine était le grand lieu de conspiration d'Europe, «plaque tournante du terrorisme international» selon l'expression de J.-M. Constant ${ }^{48}$. Le carnaval de 1580 avait réuni à Nancy des chefs de condottiere prêts à entrer dans le

${ }^{45}$ J.-J. Soons, Jeanne d'Arc au théâtre, étude sur la plus ancienne tragédie, suivie d'une liste chronologique des æuvres dramatiques dont Jeanne d'Arc a fourni le sujet en France de 1890 à 1926, Purmerend, 1929, p. 75-129 et la synthèse p. 132-133.

${ }^{46}$ L'histoire tragique..., acte IV, scène 2 : «Mais vous me demandé, s'il peust estre possible/ Que je puisse commettre aucun forfait visible/ Apres ces visions : je m'en rapporte à Dieu ».

${ }^{47} \mathrm{~J}$. De Haldat, Histoire tragique de Jeanne d'Arc en cinq actes et en vers par le père Fronton du Duc, Nancy, 1847. C $C^{\text {te }}$ de Puymaigre, Jeanne d'Arc au théâtre, 1439-1890, Paris 1890, p. 9-18. L'auteur trouve à Fronton des «qualités littéraires estimables ».

${ }^{48}$ J.-M. Constant, Les Guise, Paris, 1984, p. 112. 
royaume de France, autour de Jean Casimir de Pologne. Qui serait donc en Lorraine la «Jeanne» du roi Henri III ? Quel Guise? La question mérite d'être posée, tout comme celle d'une éventuelle commande ducale au jeune professeur de rhétorique de la toute jeune université mussipontine, lequel ne pouvait guère refuser de plonger pour la cause princière dans la bibliothèque de son établissement et fait dire à René par Charles VII :

Mon frère, je connois ce que j'ai toujours cru

Et cette opinion ne m'a jamais déçu,

Que toujours vous portez à la maison de France

Loyalle affection ; car d'elle a pris naissance

La votre aussi jadis [...]. (III, 2).

L'érudition lorraine trouva un écho, assez inattendu mais des plus honorables, à Louvain en 1629 quand Nicolas Vernulz (1583-1649), professeur d'éloquence à l'Université des Pays-Bas, composa une nouvelle tragédie, cette fois en latin, pour célébrer la mémoire de la Pucelle. Il peut s'agir d'une réponse à Shakespeare $(\dagger 1616)$, car la première édition des œuvres shakespeariennes, The first folio publié par John Heminge et Henry Condell, date de 1623. Rien ne le prouve, sinon l'orthodoxie parfaitement française et royale de l'œuvre. Vernulz voulait peutêtre montrer qu'on pouvait faire mieux que les prétentieux vers français de la Jeanne Darques de Virey des Graviers (1600) où, parmi les plus étonnants, se comptait la plainte de Jeanne condamnée :

Je ne craignois en rien les flêches de la mort ;

Mais, las, quand je me voys condamner a grand tort,

Je ne puis retenir les cours des deux fontaines

Qui roullent de mes yeux en ses humides plaines ${ }^{49}$.

Vernulz indique dans son introduction sa source fondamentale qui n'est autre que la compilation d'Hordal ${ }^{50}$. La dédicace n'est pas indifférente : l'œuvre est adressée au cardinal de Richelieu, le héros qui vient de forcer la Rochelle à capituler $\left(1^{\mathrm{er}}\right.$ novembre 1628). Le vainqueur d'ennemis du royaume soutenus par les Anglais est bien une nouvelle Jeanne d'Arc. Encore une fois tout en répondant à un contexte politique précis, la tragédie n'en est pas moins une fort belle œuvre, d'une bonne tenue dramatique et historique, écrite dans une langue classique fluide. Sa structure est nette : les cinq actes se terminent chacun par un assez long choeur (jeunes filles, soldats français puis anglais, peuple de Rouen) qui permet de changer de décor, car cette tragédie n'obéit pas aux trois unités. On note la présence en toutes circonstances d'un commentateur en la personne d'un vieillard, doué d'ubiquité et doté d'une langue bien pendue, qui interpelle aussi bien le roi Charles que Bedford, originalité qui ne paraît plus aujourd'hui aussi incongrue qu' au siècle dernier. Plantant le décor

${ }^{49}$ Virey des Graviers, Tragédie de Jeanne Darques...., op. cit., p. 42.

${ }^{50}$ Nicolas de Vernulz, Jeanne d'Arc, Tragédie latine..., op. cit., p. 4 : Ex variis incorrupta fida authoribus [...] quos fere omnes in sua de hac Puella historia complexus est Joannes Hordal, I[n]-V[troque], doctor ac Professor ducisque Lotharinga consiliarius. 
avant l'action et présentant les personnages dans leur position authentique, Vernulz fait rappeler par Charles de Bourbon (devenu plus tard le duc Charles $\mathrm{I}^{\mathrm{er}}$ ) et les futurs compagnons de Jeanne, Culan et Gilles de Rais, l'urgence de délivrer Orléans du siège par les Anglais. Jeanne commence ensuite son épopée, accompagnée de son écuyer Poulengy. Le chancelier Regnault de Chartres est d'abord réticent, comme il se doit, puis accepte de tenter l'aventure. De leur côté, les Anglais, Suffolk, Glasdale (soit ici Glacidas) et Talbot manient d'abord l'ironie puis sont remplis de terreur, devant l'élan des Français et la délivrance d'Orléans :

\author{
Talbotus \\ State, qua causa est via! \\ Qui vos! Puella sternit, ah! Sternit manu \\ Nostras cohortes; arma! Miles hàc cito \\ Arma! Miles! \\ Johanna \\ Sequere me, miles, ducem; \\ Inter hac in urbem est \\ Milites \\ Cedite Angli, cedite \\ Vincit Puella ; cedite Angli ; cedite.
}

Le roi Charles entreprend, non sans avoir tergiversé, la marche vers la Champagne, et l'on ne nous épargne aucune des villes qui se soumirent lors de la chevauchée du sacre. À Reims, Jeanne et ses frères assistent au serment royal, sont anoblis et reçoivent leurs armoiries (une épée couronnée entre deux lys) dûment décrites :
Carolus
Et te et parentes et tuos fratres creo,
Totamque gentem; decoris hujus nobilis
Signum sit ensis lilium duplex, simul
Diadema gladii cuspidem amplectens tui.
Ventura noscant tempora et colant decus.

La capture de Jeanne à Compiègne est saluée par une explosion de joie anglaise, une fois encore «à l'antique» :
Io Puellam vicimus,
Quce sola Francorum manus,
Quce sola Francorum salus,
Quae sola Francorum decus,
Io! Puella vicimus ;
Angli triumphent, vicimus!

Le rôle principal échoit ensuite au duc de Bedford, qui s'oppose à Talbot. Le premier - soutenu par Suffolk - veut exécuter Jeanne sans tarder, car la prise de la Pucelle est un signe divin en la faveur de la cause anglaise, le second veut appliquer 
les lois de la guerre. Bedford trouve dans le vêtement masculin de la Pucelle le moyen de condamner Jeanne par le tribunal, qu'il présidera lui-même. C'est l'entorse majeure de la pièce à l'histoire - il n'y a pas de jugement par l'Église. Jeanne quitte la scène pour le supplice, le choeur lui prédit une reconnaissance éternelle et une cérémonie annuelle au monument qui remplacera ses cendres jetées au fleuve :

Et depexo crine quotannis

Dabimus moeste tumulo lacrymas

Dabimus novos tumulo flores.

Malheureusement cette pièce, perdue au milieu d'une dizaine d'autres tragédies latines de Vernulz demeurées encore plus confidentielles, n'a pas obtenu le retentissement qu'elle aurait certainement mérité, ni le privilège d'une mise en vers français contemporaine qui n'eût pas été forcément détestable. Cependant elle fut exhumée dans les années 1880 par le comte de Puymaigre $^{51}$, traduite à Orléans par André De Latour et éditée chez le libraire Herluison en 1890.

Le drame de Vernulz nous offre une scène remarquable. Au moment de partir avec Poulengy, Jeanne se tourne vers son pays et lui fait ses adieux :

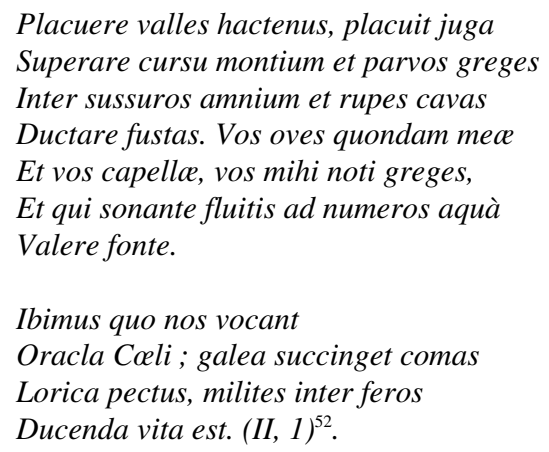

Si le thème des adieux au pays que l'on quitte sans retour n'est pas neuf, l'origine de son application à Jeanne d'Arc se trouve sans doute au chapitre 59 de la Chronique de la Pucelle. Après le sacre, le 14 août 1429, Jeanne qui se trouvait près de Dammartin et souhaitait mourir au sein de ce peuple en liesse par le retour du roi, évoque alors son pays natal : «je voudrois bien qu'il [Messire Dieu] voulût me faire ramener auprès mes pères et mères, et garder leurs brebis et bestail, et faire ce que je soulois faire $»^{53}$.

${ }^{51} \mathrm{C}^{\mathrm{te}}$ de Puymaigre, Jeanne d'Arc au théâtre, op. cit., pp. 27-33.

${ }^{52}$ « J'aimais jusqu'ici les vallées, j'aimais à franchir la cime des montagnes et à conduire de ma houlette mes humbles troupeaux le long des fleuves murmurants et au pied des rochers. Brebis autrefois miennes, et vous mes chèvres, et vous troupeaux connus de moi, et vous dont les ondes coulaient avec un si harmonieux murmure, adieu, ô fontaines [...] J'irai où m'appellent les oracles du ciel. Le casque couvrira ma chevelure, la cuirasse ma poitrine; il me faudra vivre entre les rudes soldats », traduction de A. De Latour, op. cit., p. 46-47.

${ }^{53}$ Chronique de la Pucelle, éd. Vallet de Viriville, Paris, Delahays, 1869, p. 326. 
Il y a plus d'un siècle que le rapprochement a été fait par M. Bailly entre des vers de la première Eglogue de Virgile, le texte de Vernulz et les stances de Jeanne à la fin du prologue de la Jungfrau von Orleans de Schiller :

Lebt Wohl, ihr Berge, ihr geliebten Triften

Ihr traulich stillen Täler, lebet wohl !

Johanna wird nun nicht mehr auf euch wandeln,

Johanna sagt euch ewig Lebewohl ${ }^{54}$.

Rapprochement opéré dans édition scolaire de la tragédie allemande ${ }^{55}$, presque contemporaine de la première traduction française - et orléanaise - de Vernulz : Schiller, commente Bailly «suit de près pour le fonds de ces cinq stances un monologue d'une Johanna Darcia, tragédie latine, écrite, il y avait près de deux siècles, par un certain Nicolaus Vernulæus». Le commentateur cite ensuite les vers que Schiller «a imités de son compatriote » et conclut : «ce devoir d'écolier est devenu l'admirable monologue qui est sinon dans toutes les mémoires, du moins dans toutes les anthologies ». Le mépris envers le texte de Vernulz me semble largement injustifié tant la cascade d'imitations révèle en vérité le talent propre de chaque auteur.

Il me paraît évident que Charles Péguy, fort au courant des nouveautés éditées dans sa ville, a connu ces deux textes; en bon élève classique il a goûté une partie des qualités littéraires du XVII ${ }^{\mathrm{e}}$ siècle latin et y a peut-être trouvé, entrant en concurrence vraisemblablement volontaire avec le poète allemand, l'idée de ces adieux au pays d'enfance qui sont parmi les lignes les plus célèbres de sa Jeanne $d^{\prime}$ Arc de $1897^{56}$ et peut-être de son œuvre tout entière : "Adieu Meuse endormeuse et douce à mon enfance... ». Le génie du poète a, à son tour, magnifiquement transposé et interprété les allitérations latines dans une page lyrique parfaitement réussie.

Malgré les efforts d'Hordal, et un peu plus tard des sérieuses recherches de textes de Denis Godefroy, l'image historique de Jeanne d'Arc se brouille de plus en plus au XVII ${ }^{\mathrm{e}}$ siècle, même pour le public cultivé. Godefroy avait pourtant accompli un travail considérable en faisant découvrir à l'occasion d'un recueil concernant Charles VII, le Journal d'un Bourgeois de Paris, Jean Chartier, les Chroniques du roi Charles VII du Héraut Berry, la Chronique de la Pucelle et de nombreuses pièces touchant au roi et à l'histoire de Jeanne d' $\mathrm{Arc}^{57}$. La vérité intrinsèque du personnage n'intéressait pas les lecteurs contemporains qui ne trouvaient sans doute pas là assez d'inconnu pour attiser leur imagination. On ne peut guère penser autrement à la lecture des œuvres qui firent au milieu du siècle quelque bruit dans le monde des lettres et des salons.

${ }^{54}$ Schiller, La Pucelle d'Orléans (Die Jungfrau von Orleans), éd. et trad. W. Thomas, Paris, 1932 , prologue, scène IV, p. 16 : «Adieu monts, adieu paysages aimés, vous, vallées familières et paisibles, adieu! Jeanne désormais ne vaguera plus sur vous, Jeanne vous dit à jamais adieu. »

${ }^{55}$ Schiller, Jeanne d'Arc. Tragédie. Texte allemand, publié avec un argument, une notice littéraire, des éclaircissements et des notes par M. Bailly, Paris, Hachette, 1886, p. 80-81, n. 1. ${ }^{56}$ [Marcel et Pierre Baudoin] Jeanne d'Arc, drame en trois pièces, Domremy, les Batailles, Rouen, Paris, La librairie socialiste, 1897, 752 p. non numérotées.

${ }^{57}$ D. Godefroy, Histoire de Charles VII roi de France par Jean Chartier [...] qui contient les choses les plus remarquables depuis l'an 1422 jusqu'en 1461. Mise en lumière et enrichie de plusieurs titres, mémoires, traitez... Paris, Sébastien Cramoisy, 1661. 
L'abbé d'Aubignac, François Hedelin, se lança dans une tragédie en prose, presque immédiatement mise en vers par la Mesnardière. On n'en dirait plus rien aujourd'hui, si elle n'avait été la première à ramasser l'action en vingt-quatre heures du procès, ce qui fut par la suite le propos de Carl Dreyer et de Bertold Brecht. La Pucelle d'Orléans travestit l'Histoire en y faisant entrer le romanesque et les coups de théâtre, mais d'une toute autre façon que dans le Henry VI de Shakespeare.

Warwick, le geôlier de la Pucelle à Rouen est, au grand désespoir de la comtesse sa femme, amoureux de Jeanne; il essaie de la faire évader, elle refuse et l'amour et la liberté. Jeanne marche au supplice malgré un retournement spectaculaire de la comtesse qui sombre dans la folie en demandant la grâce de sa rivale, tandis que, comme l'avait prédit l'héroïne, le procureur d'Estivet est chassé par la foule, qu'un des clercs persécuteurs est atteint subitement de la peste et que Cauchon (ici un bizarre et typographique Canchon!) est foudroyé d'apoplexie sur scène. Parmi les étranges discours que l'on entend, une assez longue tirade de Jeanne adressée au duc de Bedford sur les vertus de la loi salique prédit aux Anglais le «joug d'une femme altière et cruelle et puissante» (II, 2), évocation où l'on doit voir se profiler la grande Elisabeth. Mais en réalité dans ces tragédies baroques, Jeanne continue à perdre son caractère et sa force sous les plumes en panache qui encombrent sa tête, au point qu'un des personnages déclare non sans justesse : «et quoy que ridicule elle estonne pourtant ${ }^{58}$. Le véritable héros, l'amoureux de tragédie sans nuances toutefois, est ici un improbable comte de Warwick, transi.

Que Jeanne passe au second plan des œuvres qui lui sont consacrées est encore plus net avec Chapelain, et sa trop célèbre Pucelle ou la France délivrée. Nous ne nous étendrons pas sur les alexandrins tirés à la ligne, souvent mauvais et parfois ridicules, autant que ceux de Virey des Graviers, et dont à juste titre Boileau fit de peu charitables gorges chaudes. Dans le Dialogue des héros de romans, la place de Jeanne au rang des personnages imaginaires souligne le glissement de l'histoire à la fiction : c'est une virago qui s'exprime en vers fort piteux, cruellement empruntés à Chapelain par l'impitoyable censeur ${ }^{59}$. La Pucelle eut cependant du succès et nombreuses furent les éditions de tous formats. Les treize illustrations de Vignon, gravées par Abraham Bosse, qu'offraient les plus précieux exemplaires (un frontispice et la première page de chaque chant: Jeanne Bergère, Jeanne au sacre, Jeanne au siège de Paris, Jeanne à Compiègne etc...) servirent de modèles à une série de cartons d'importantes tapisseries réalisées en assez grand nombre, peut-être à Aubusson. Certaines de ces tentures sont encore visibles aujourd'hui, attestant la durable popularité du thème ${ }^{60}$. Mais le personnage éponyme de la Pucelle ne semble pas avoir inspiré le poète qui, pour des raisons de clientélisme envers les Longueville, avait de son propre aveu fait de Dunois, leur ancêtre, son héros véritable, "mon Ulysse». Il a multiplié des personnages qui n'avaient rien à faire là, Agnès Sorel

${ }^{58}$ [La Mesnardière], La Pucelle d'Orléans, 1642, p. 83, acte V, scène 1.

${ }^{59} \mathrm{~N}$. Boileau, Dialogue des héros de roman, [1688], Euvres complètes, éd. A. Adam et F. Escal, Paris, 1966 (La Pléiade), p. 479-482.

${ }^{60}$ Plusieurs séries incomplètes de ces tentures sont conservées à Orléans dont une au Musée des Beaux Arts, une autre à la Maison de Jeanne d'Arc. Une série se trouvait à Comblat (Cantal), une quatrième fut volée en 1976 au château d'Ussé (Indre-et-Loire). Les illustrations de Vignon étaient interprétées en fonction des commandes des clients et des dimensions accordées aux pièces à réaliser. 
entre autres, et réduit délibérément Jeanne a n'être simplement que «la Pallas de [son] Ulysse $»^{61}$.

La figure de la Pucelle n'en prend pas moins dans cette interminable épopée une forme nouvelle dont l'auteur ne mesurait certainement pas le caractère prophétique. À la lecture intégrale cette Pucelle apparaît comme une étape dans le cheminement qui devait mener à la canonisation de 1920 . Nous sommes revenus ici à la lignée des vies de femmes célèbres et de héros-modèle, dans la veine de la Légende dorée, et - déjà - de l'hagiographie.

Les années centrales du XVII ${ }^{\mathrm{e}}$ siècle marquent pour l'histoire de la sainteté de Jeanne d'Arc un épisode, tout provisoire néanmoins, de la marche vers les autels. Le Martyrologium gallicanum ${ }^{62}$ indiquait à la date du 30 mai le martyre de Jeanne sur la place du vieux marché à Rouen en mentionnant l'annulation de la condamnation: damnatio injusta sedis apostolica revocata, prorsusque abrogata fuerit. En 1647 , l'historien orléanais Symphorien Guyon évoquait à son tour la «bienheureuse Jeanne d'Arc $»^{63}$. Chapelain a franchi un pas en usant sans fard du terme «sainte ».

Jeanne parle rarement et agit peu dans le poème, mais il est question d'elle un certain nombre de fois, surtout au cours des douze premiers chants, seuls parus au $\mathrm{XVII}^{\mathrm{e}}$ siècle. Un relevé systématique des désignations sous lesquelles elle apparaît confirme la place nouvelle accordée à sa sainteté supposée. Pour ne pas se répéter à satiété, Chapelain a utilisé vingt et une manières différentes de désigner son personnage-titre, dont quatre seulement défavorables qu'il place dans la bouche des Anglais. On peut en consulter la liste et les occurrences en annexe. En regroupant noms et épithètes par grands thèmes on voit Jeanne se présenter sous trois figures: la sainte, la guerrière et la fille ou pucelle, voire «la Pucelle». La préférence est clairement donnée à la première désignation qui replace une aventure exceptionnelle dans le dessein de Dieu, mais aussi dans les nuées baroques de la condition extra mortelle. Cela autorise les contraventions aux usages de la réserve féminine et l'usage des accessoires de costume militaire à l'antique. Or la Fronde terminée, on n'appréciait plus autant les belles empanachées. En particulier à Orléans, où l'intervention intempestive de la Grande Mademoiselle ${ }^{64}$ n'avait pas laissé que de bons souvenirs.

${ }^{61}$ J. Chapelain, La Pucelle ou la France délivrée, E. de Molènes éd., Paris, 1891, p. LVII.

${ }^{62}$ Martyrologium gallicanum, Paris, André du Saussay, 1637 (cf. Lanéry d'Arc, n ${ }^{\circ} 1352$ ).

${ }^{63}$ S. Guyon, Histoire de l'église et diocèse, ville et université d'Orléans, Orléans, Paris, Maria, 1647. Table alphabétique des saints du diocèse d'Orléans.

${ }^{64}$ Le 16 mars 1652, la Grande Mademoiselle força la poterne Barentin sur le fleuve et entra triomphante dans Orléans. Une gravure partisane la montre, Minerve en jupon, terrassant Mazarin (qui n'était pas présent en réalité) sur le quai de la Loire (cf. J. Vassort, Grandeur et déclin d'Orléans, de 1600 à 1870, Histoire d'Orléans et de son terroir, dir. J. Debal, Roanne, 1982, t. II, p. 38-40). 


\section{Designations de Jeanne D’Arc dans La PuCELLE de ChaPelain}

\begin{tabular}{|l|l|l|l|l|}
\hline DESIGNATION & 1657 (12 chants) & $\%$ & Total $(24$ chants $)$ & $\%$ \\
\hline Sainte/céleste & 96 & 47,5 & 106 & 50 \\
\hline Guerrière & 35 & 17,3 & 36 & 17 \\
\hline Fille/Pucelle & 71 & 35,2 & 73 & 33 \\
\hline TOTAL & 202 & 100 & 213 & 100 \\
\hline
\end{tabular}

Certaines désignations comportant deux éléments : «sainte fille, guerrière céleste etc », se trouvent comptées deux fois.

En 1672, le révérend père Senault de l'Oratoire dans un des rares panégyriques du 8 mai orléanais conservés pour ce siècle, pensait avoir le droit d'affirmer; «proclamons là donc mille fois bienheureuse : adressons lui nos prières ${ }^{65}$. Mais il est certain que la royauté n'avait pas, alors, besoin d'une sainte laïque, d'une fille en arme, d'une "amazone céleste », descendue d'un plafond. Peut-être avions-nous là les prémisses d'une sainteté de fait, mais qui tourna vite court. N'accablons pas Chapelain en suggérant qu'il a aggravé la cause de la canonisation.

La Jeanne historique et la Jeanne spirituelle, si les deux aspects du personnage sont séparables, semblent bien marquer le pas au milieu du XVII siècle. Mezeray, dans son Abrégé Chronologique où des générations de jeunes gens apprirent ensuite l'histoire de France, exprime bien le recul officiel d'une historiographie devenue un instrument de l'absolutisme. Jeanne ne se voit pas confier l'armée, la chronologie est défaillante: Orléans y est délivrée le 12 mai, au lieu du 8, ce qui devait rendre inexplicable la date de la fête de la ville. La victoire est essentiellement remportée par les assiégés, auxquels il accorde que la Pucelle a quand même redonné courage. L'héroïne échoue devant Paris en raison de sa désobéissance et de son entêtement, «le ciel n'estant pas obligé de l'assister en ce qu'il ne luy avoit pas commandé ». L'auteur conte la capture, le jugement et la mort de Jeanne avec sécheresse, sans taire la responsabilité de «ce lambeau d'université qui estoit resté à Paris», pas plus que le mécontentement des Anglais devant une première condamnation trop douce « au pain de douleur et à l'eau d'amertume $»^{66}$.

Seul Pierre le Moyne semble avoir senti avec lucidité la grandeur de Jeanne mais aussi l'ambiguïté du personnage : «sa vertu estoit sensible et avoit un corps : elle a fait véritablement et à la campagne tout ce que celles là n'ont fait qu'en peinture et dans le cerveau de leurs autheurs ${ }^{67}$. Il est clair, cependant, que cette pensée demeure isolée et qu'à l'époque classique Jeanne d'Arc, devenue universellement un personnage, n'était plus une personne.

${ }^{65}$ E. Séjourné, La canonisation de Jeanne d'Arc. État actuel de la cause, Orléans, G. Séjourné, 1887, p. 11, n. 1.

${ }^{66}$ E. de Mezeray, Abrégé chronologique ou extraict de l'histoire de France, Paris, Thomas Jolly, 1668, t. II, p. 684-688. La première édition est de 1643-1651.

${ }^{67} \mathrm{P}$ Lemoyne, La gallerie des femmes fortes, Paris, Antoine de Sommaville, 1647, p. 304 et suivantes. « À la campagne » est à prendre ici au sens de campagne militaire. 


\section{ANNEXE}

LES DÉSIGNATIONS DE JEANNE D'ARC DANS LA PUCELLE DE

CHAPELAIN

\begin{tabular}{|c|c|c|c|}
\hline DÉSIGNATION & CHANTS I-XII & CHANTS XII-XXIV & TOTAL \\
\hline Amazone du ciel & 1 & - & 1 \\
\hline Bergère & 1 & - & 1 \\
\hline Céleste guerrière & 1 & - & 1 \\
\hline Effrontée & 1 & - & 1 \\
\hline Enchanteresse & 1 & - & 1 \\
\hline Faible sainte & 1 & - & 1 \\
\hline Fille & 31 & - & 31 \\
\hline Fille céleste & 2 & - & 2 \\
\hline Fille des cieux & 3 & - & 3 \\
\hline Fille divine & 1 & 1 & 2 \\
\hline Fille magnanime & 1 & - & 1 \\
\hline Française Bellone & 1 & - & 1 \\
\hline Héroïne & 3 & - & 3 \\
\hline La guerrière & 26 & 1 & 27 \\
\hline Pucelle & 29 & 3 & 32 \\
\hline Sainte & 78 & 9 & 87 \\
\hline Sainte bergère & 2 & - & 2 \\
\hline Sainte fille & 5 & - & 5 \\
\hline Sainte guerrière & 2 & - & 2 \\
\hline Sorcière & 4 & 1 & 5 \\
\hline Terrible guerrière & 1 & - & 1 \\
\hline Traîtresse & 1 & - & 1 \\
\hline TOTAL & 203 & 15 & 218 \\
\hline
\end{tabular}

On notera la place fort réduite accordée à l'héroïne éponyme du poème dans la partie restée inédite jusqu' au XIX ${ }^{\mathrm{e}}$ siècle ! 